\title{
Engel Series and Cohen-Egyptian Fraction Expansions
}

\author{
Vichian Laohakosol, ${ }^{1}$ Tuangrat Chaichana, ${ }^{2,3}$ \\ Jittinart Rattanamoong, ${ }^{2,3}$ and Narakorn Rompurk Kanasri ${ }^{4}$ \\ ${ }^{1}$ Department of Mathematics, Kasetsart University, Bangkok 10900, Thailand \\ ${ }^{2}$ Department of Mathematics, Faculty of Science, Chulalongkorn University, Bangkok 10330, Thailand \\ ${ }^{3}$ The Centre of Excellence in Mathematics, CHE, Si Ayutthaya Road, Bangkok 10400, Thailand \\ ${ }^{4}$ Department of Mathematics, Khon Kaen University, Khon Kaen 40002, Thailand
}

Correspondence should be addressed to Vichian Laohakosol, fscivil@ku.ac.th

Received 11 September 2009; Accepted 2 December 2009

Recommended by Stéphane Louboutin

Two kinds of series representations, referred to as the Engel series and the Cohen-Egyptian fraction expansions, of elements in two different fields, namely, the real number and the discrete-valued non-archimedean fields are constructed. Both representations are shown to be identical in all cases except the case of real rational numbers.

Copyright ( 2009 Vichian Laohakosol et al. This is an open access article distributed under the Creative Commons Attribution License, which permits unrestricted use, distribution, and reproduction in any medium, provided the original work is properly cited.

\section{Introduction}

It is well known [1] that each nonzero real number can be uniquely written as an Engel series expansion, or ES expansion for short, and an ES expansion represents a rational number if and only if each digit in such expansion is identical from certain point onward. In 1973, Cohen [2] devised an algorithm to uniquely represent each nonzero real number as a sum of Egyptian fractions, which we refer to as its Cohen-Egyptian fraction (or CEF) expansion. Cohen also characterized the real rational numbers as those with finite CEF expansions. At a glance, the shapes of both expansions seem quite similar. This naturally leads to the question whether the two expansions are related. We answer this question affirmatively for elements in two different fields. In Section 2, we treat the case of real numbers and show that for irrational numbers both kinds of expansion are identical, while for rational numbers, their ES expansions are infinite, periodic of period 1, but their CEF expansions always terminate. In Section 3, we treat the case of a discrete-valued non-archimedean field. After devising ES and CEF expansions for nonzero elements in this field, we see immediately that both expansions are identical. In Section 4, we characterize rational elements in three different non-archimedean fields. 


\section{The Case of Real Numbers}

Recall the following result, see, for example, Kapitel IV of [1], which asserts that each nonzero real number can be uniquely represented as an infinite ES expansion and rational numbers have periodic ES expansions of period 1.

Theorem 2.1. Each $A \in \mathbb{R} \backslash\{0\}$ is uniquely representable as an infinite series expansion, called its Engel series (ES) expansion, of the form

$$
A=a_{0}+\sum_{n=1}^{\infty} \frac{1}{a_{1} a_{2} \cdots a_{n}}
$$

where

$$
a_{0}=\left\{\begin{array}{ll}
{[A],} & \text { if } A \notin \mathbb{Z}, \\
A-1 & \text { if } A \in \mathbb{Z},
\end{array} \quad a_{1} \geq 2, a_{n+1} \geq a_{n} \quad(n \geq 1) .\right.
$$

Moreover, $A \in \mathbb{Q}$ if and only if $a_{n+1}=a_{n}(\geq 2)$ for all sufficiently large $n$.

Proof. Define $A_{1}=A-a_{0}$, then $0<A_{1} \leq 1$. If $A_{n} \neq 0(n \geq 1)$ is already defined, put

$$
\begin{aligned}
& a_{n}=1+\left[\frac{1}{A_{n}}\right], \\
& A_{n+1}=a_{n} A_{n}-1 .
\end{aligned}
$$

Observe that $a_{n}$ is the least integer $>1 / A_{n}$ and

$$
\frac{1}{a_{n}}<A_{n} \leq \frac{1}{a_{n}-1}
$$

We now prove the folowing.

Claim. We have $0<\cdots \leq A_{n+1} \leq A_{n} \leq \cdots \leq A_{2} \leq A_{1} \leq 1$.

Proof of the Claim. First, we show that $A_{n}>0$ for all $n \geq 1$ by induction. If $n=1$, we have seen that $A_{1}>0$. Assume now that $A_{n}>0$ for $n \geq 1$. By (2.3), we see that $a_{n} \in \mathbb{N}$. Since

$$
A_{n+1}=a_{n} A_{n}-1=\left(A_{n}-\frac{1}{a_{n}}\right) a_{n}
$$

and $1 / a_{n}<A_{n}$, we have $A_{n+1}>0$. If there exists $k \in \mathbb{N}$ such that $A_{k+1}>A_{k}$, then

$$
a_{k} A_{k}-1=A_{k+1}>A_{k}
$$

and so $a_{k}-1>1 / A_{k}$, contradicting the minimal property of $a_{n}$ and the Claim is proved. 
From the Claim and (2.3), we deduce that $a_{1} \geq 2$ and $a_{n+1} \geq a_{n}(n \geq 1)$. Iterating (2.4), we get

$$
A_{1}=\frac{1}{a_{1}}+\frac{1}{a_{1} a_{2}}+\cdots+\frac{1}{a_{1} a_{2} \cdots a_{n}}+\frac{A_{n+1}}{a_{1} a_{2} \cdots a_{n}} .
$$

To establish convergence, let

$$
B_{n}=\frac{1}{a_{1}}+\frac{1}{a_{1} a_{2}}+\cdots+\frac{1}{a_{1} a_{2} \cdots a_{n}} \quad(n \geq 1) .
$$

Since $A_{n}>0$ and $a_{n} \in \mathbb{N}$ for all $n \geq 1$, the sequence of real numbers $\left(B_{n}\right)$ is increasing and bounded above by $A_{1}$. Thus, $\lim _{n \rightarrow \infty} B_{n}$ exists and so

$$
\frac{1}{a_{1} a_{2} \cdots a_{n}} \longrightarrow 0 \quad(n \longrightarrow \infty)
$$

By the Claim,

$$
0<\frac{A_{n+1}}{a_{1} a_{2} \cdots a_{n}} \leq \frac{1}{a_{1} a_{2} \cdots a_{n}} \longrightarrow 0 \quad(n \longrightarrow \infty)
$$

showing that any real number has an ES expansion. To prove uniqueness, assume that we have two infinite such expansions such that

$$
a_{0}+\sum_{n=1}^{\infty} \frac{1}{a_{1} a_{2} \cdots a_{n}}=b_{0}+\sum_{n=1}^{\infty} \frac{1}{b_{1} b_{2} \cdots b_{n}},
$$

with the restrictions $a_{0} \in \mathbb{Z}, a_{1} \geq 2, a_{n+1} \geq a_{n}(n \geq 1)$ and the same restrictions also for the $b_{n}{ }^{\prime}$ s. From the restrictions, we note that

$$
0<A_{1}:=\sum_{n=1}^{\infty} \frac{1}{a_{1} a_{2} \cdots a_{n}} \leq 1
$$

If $A_{1}=1$, then by (2.12) we also have $\sum_{n \geq 1} 1 / b_{1} b_{2} \cdots b_{n}=1$, forcing $a_{0}=b_{0}$. If $0<A_{1}<1$, then (2.12) shows that $0<\sum_{n \geq 1} 1 / b_{1} b_{2} \cdots b_{n}<1$, forcing again $a_{0}=b_{0}$. In either case, cancelling out the terms $a_{0}, b_{0}$ in (2.12) we get

$$
A_{1}:=\sum_{n=1}^{\infty} \frac{1}{a_{1} a_{2} \cdots a_{n}}=\sum_{n=1}^{\infty} \frac{1}{b_{1} b_{2} \cdots b_{n}} .
$$

Since $a_{n+1} \geq a_{n}$, then

$$
a_{1} A_{1}-1=\frac{1}{a_{2}}+\frac{1}{a_{2} a_{3}}+\frac{1}{a_{2} a_{3} a_{4}}+\cdots \leq \frac{1}{a_{1}}+\frac{1}{a_{1} a_{2}}+\frac{1}{a_{1} a_{2} a_{3}}+\cdots=A_{1},
$$


so $0<a_{1}-1 / A_{1} \leq 1$. But there is exactly one integer $a_{1}$ satisfying these restrictions. Thus, $a_{1}=b_{1}$. Cancelling out the terms $a_{1}$ and $b_{1}$ in (2.14) and repeating the arguments we see that $a_{i}=b_{i}$ for all $i$.

Concerning the rationality characterization, if its ES expansion is infinite periodic of period 1 , it clearly represents a rational number. To prove its converse, let $A=a / b \in \mathbb{Q} \backslash\{0\}$. Since

$$
A_{1}=A-a_{0}=\frac{a-b a_{0}}{b}
$$

we see that $A_{1}$ is a rational number in the interval $(0,1]$ whose denominator is $b$. In general, from (2.4), we deduce that each $A_{n}(n \geq 1)$ is a rational number in the interval $(0,1]$ whose denominator is $b$. But the number of rational numbers in the interval $(0,1]$ whose denominator is $b$ is finite implying that there are two least suffixes $h, k \in \mathbb{N}$ such that $A_{h+k}=A_{h}$. Thus, by (2.3), we have $a_{h+k}=a_{h}$. From (2.2), we know that the sequence $\left\{a_{n}\right\}$ is increasing. We must then have $k=1$ and the assertion follows.

Remarks. In passing, we make the following observations.

(a) For $n \geq 1$, we have

$$
a_{n+1}=a_{n} \Longleftarrow A_{n+1}=A_{n} \Longleftrightarrow a_{n} A_{n}-1=A_{n} \Longleftrightarrow a_{n}=1+\frac{1}{A_{n}} \Longleftrightarrow \frac{1}{A_{n}} \in \mathbb{Z} .
$$

(b) If $A \in \mathbb{Q}^{c}$, then $A_{n} \in \mathbb{Q}^{c}$ and so $1 / A_{n} \notin \mathbb{Q}$ for all $n \geq 1$.

(c) If $A \in \mathbb{Z}$, then its ES expansion is

$$
A=A-1+\frac{1}{2}+\frac{1}{2^{2}}+\frac{1}{2^{3}}+\cdots
$$

To construct a Cohen-Egyptian fraction expansion, we proceed as in [2] making use of the following lemma.

Lemma 2.2. For any $y \in(0,1)$, there exist a unique integer $n \geq 2$ and a unique $r \in \mathbb{R}$ such that

$$
1=n y-r, \quad 0 \leq r<y
$$

Proof. Let $n=\lceil 1 / y\rceil \in \mathbb{N}$ and $r=n y-1$. Put $\langle 1 / y\rangle:=n-1 / y \in[0,1)$ and so

$$
r=n y-1=y\left\langle\frac{1}{y}\right\rangle \in[0, y)
$$

To prove uniqueness, assume $n y-r=1=m y-s$ so that

$$
1+\frac{1}{y}>n=\frac{1+r}{y} \geq \frac{1}{y}
$$


Since there is only one integer with this property, we deduce $n=m$ and consequently, $r=s$ proving the lemma.

Theorem 2.3. Each $A \in \mathbb{R} \backslash\{0\}$ is uniquely representable as a CEF expansion of the form

$$
A=n_{0}+\sum_{k=1}^{\infty} \frac{1}{n_{1} n_{2} \cdots n_{k}}
$$

subject to the condition

$$
n_{0} \in \mathbb{Z}, \quad n_{1} \geq 2, \quad n_{k+1} \geq n_{k} \quad(k \geq 1)
$$

and no term of the sequence appears infinitely often. Moreover, each CEF expansion terminates if and only if it represents a rational number.

Proof. To construct a CEF expansion for $A \in \mathbb{R} \backslash\{0\}$, define

$$
r_{0}=A-n_{0} \in[0,1)
$$

If $r_{0}=0$, then the process stops and we write $A=n_{0}$. If $r_{0} \neq 0$, by Lemma 2.2, there are unique $n_{1} \in \mathbb{N}$ and $r_{1} \in \mathbb{R}$ such that

$$
1=n_{1} r_{0}-r_{1}, \quad 0 \leq r_{1}<r_{0}, n_{1} \geq 2
$$

Thus,

$$
A=n_{0}+r_{0}=n_{0}+\frac{1}{n_{1}}+\frac{r_{1}}{n_{1}}
$$

If $r_{1}=0$, then the process stops and we write $A=n_{0}+1 / n_{1}$. If $r_{1} \neq 0$, by Lemma 2.2, there are $n_{2} \in \mathbb{N}$ and $r_{2} \in \mathbb{R}$ such that

$$
1=n_{2} r_{1}-r_{2}, \quad 0 \leq r_{2}<r_{1}, n_{2} \geq n_{1}
$$

the last inequality being followed from $n_{1}=\left\lceil 1 / r_{0}\right\rceil, n_{2}=\left\lceil 1 / r_{1}\right\rceil$, and $r_{1}<r_{0}$. Observe also that

$$
A=n_{0}+\frac{1}{n_{1}}+\frac{1}{n_{1} n_{2}}+\frac{r_{2}}{n_{1} n_{2}}
$$

Continuing this process, we get

$$
A=n_{0}+\frac{1}{n_{1}}+\frac{1}{n_{1} n_{2}}+\cdots+\frac{1}{n_{1} n_{2} \cdots n_{k}}+\frac{r_{k}}{n_{1} n_{2} \cdots n_{k}}
$$


with

$$
1=n_{i} r_{i-1}-r_{i}, \quad 1>r_{i-1}>r_{i} \geq 0,2 \leq n_{i} \leq n_{i+1}(i=1,2, \ldots) .
$$

If some $r_{k}=0$, then the process stops, otherwise the series convergence follows at once from

$$
\left|\frac{r_{k}}{n_{1} n_{2} \cdots n_{k}}\right| \longrightarrow 0 \quad(k \rightarrow \infty)
$$

To prove uniqueness, let

$$
n_{0}+\sum_{k=1}^{\infty} \frac{1}{n_{1} n_{2} \cdots n_{k}}=A=m_{0}+\sum_{k=1}^{\infty} \frac{1}{m_{1} m_{2} \cdots m_{k}}
$$

with the restrictions (2.23) on both digits $n_{i}$ and $m_{j}$. Now

$$
\sum_{k \geq 1} \frac{1}{n_{1} n_{2} \cdots n_{k}} \leq \sum_{k \geq 1} \frac{1}{2^{k}}=1
$$

It is clear that the restrictions (2.23) imply the strict inequality in (2.33). This also applies to the right-hand sum in (2.32). Equating integer and fractional parts in (2.32), we get

$$
n_{0}=m_{0}, \quad \sum_{k=1}^{\infty} \frac{1}{n_{1} n_{2} \cdots n_{k}}=\sum_{k=1}^{\infty} \frac{1}{m_{1} m_{2} \cdots m_{k}}=: w, \text { say. }
$$

Since $n_{k+1} \geq n_{k}$, then

$$
n_{1} w-1=\frac{1}{n_{2}}+\frac{1}{n_{2} n_{3}}+\frac{1}{n_{2} n_{3} n_{4}}+\cdots \leq \frac{1}{n_{1}}+\frac{1}{n_{1} n_{2}}+\frac{1}{n_{1} n_{2} n_{3}}+\cdots=w
$$

so $0<n_{1}-1 / w \leq 1$. But there is exactly one integer $n_{1}$ satisfying these restrictions. Then $n_{1}=m_{1}$ and

$$
\sum_{k \geq 2} \frac{1}{n_{2} \cdots n_{k}}=\sum_{k \geq 2} \frac{1}{m_{2} \cdots m_{k}}
$$

Proceeding in the same manner, we conclude that $n_{i}=m_{i}$ for all $i$.

Finally, we look at its rationality characterization. If $A \in \mathbb{Q}$, then $r_{0} \in \mathbb{Q}$, say $r_{0}:=p / q$, where $p, q \in \mathbb{N}$. From (2.30), we see that each $r_{i}$ is a rational number whose denominator is $q$. Using this fact and the second inequality condition in (2.30), we deduce that $r_{j}=0$ for some $j \leq p$, that is, the expansion terminates. On the other hand, it is clear that each terminating 
CEF expansion represents a rational number. Now suppose that $A$ is irrational and there is a $j$ and integer $n$ such that $n_{i}=n$ for all $i \geq j$. Then

$$
A=n_{0}+\sum_{k=1}^{j} \frac{1}{n_{1} n_{2} \cdots n_{k}}+\frac{1}{n_{1} n_{2} \cdots n_{j}} \sum_{k=1}^{\infty} \frac{1}{n^{k}} .
$$

Since $\sum_{k \geq 1} 1 / n^{k}=1 /(n-1)$, it follows that $A$ is rational, which is impossible.

The connection and distinction between ES and CEF expansions of a real number are described in the next theorem.

Theorem 2.4. Let $A \in \mathbb{R} \backslash\{0\}$ and the notation be as set out in Theorems 2.1 and 2.3.

(i) If $A \in \mathbb{Q}$, then its ES expansion is infinite periodic of period 1, while its CEF expansion is finite. More precisely, for $A \in \mathbb{Q} \backslash \mathbb{Z}$, let its $E S$ and $C E F$ expansions be, respectively,

$$
A=a_{0}+\sum_{n=1}^{\infty} \frac{1}{a_{1} a_{2} \cdots a_{n}}=n_{0}+\sum_{k=1}^{\infty} \frac{1}{n_{1} n_{2} \cdots n_{k}} .
$$

If $m$ is the least positive integer such that $1 / A_{m} \in \mathbb{Z}$, then

$$
a_{0}=n_{0}, \quad a_{1}=n_{1}, \ldots, \quad a_{m-1}=n_{m-1}, \quad a_{m}=n_{m}+1, \quad a_{m}=a_{m+i} \quad(i \geq 1),
$$

and the digits $n_{i}$ terminate at $n_{m}$.

(ii) If $A \in \mathbb{R} \backslash \mathbb{Q}$, then its ES and its CEF expansions are identical.

Proof. Both assertions follow mostly from Theorems 2.1, 2.3, and Remark (b) except for the result related to the expansions in (2.38) which we show now.

Let $A \in \mathbb{Q} \backslash \mathbb{Z}$ and let $m$ be the least positive integer such that $1 / A_{m} \in \mathbb{Z}$. We treat two seperate cases.

Case $1(m=1)$. In this case, we have $1 / A_{1} \in \mathbb{Z}$ and $a_{1}=1+\left[1 / A_{1}\right]=1+1 / A_{1}$. Since $r_{0}=A-n_{0}=A-[A]=A-a_{0}=A_{1}$, we get $n_{1}=1 / A_{1}$ and so $a_{1}=n_{1}+1$. We have $r_{1}=n_{1} r_{0}-1=0$, and so the CEF expansion terminates. On the other hand, by Remark (a) after Theorem 2.1, we have $a_{1}=a_{i}(i \geq 2)$.

Case $2(m>1)$. Thus, $1 / A_{1} \notin \mathbb{Z}$ and $A_{1}=r_{0}$. By Lemma 2.2, we have $a_{1}=n_{1}$. For $1 \leq i \leq m-2$, assume that $A_{i}=r_{i-1}$ and $a_{i}=n_{i}$. Then

$$
A_{i+1}=a_{i} A_{i}-1=n_{i} r_{i-1}-1=r_{i}
$$

Since $1 / A_{i+1} \notin \mathbb{Z}$, again by Lemma $2.2, a_{i+1}=n_{i+1}$. This shows that $a_{1}=n_{1}, \ldots, a_{m-1}=n_{m-1}$. Since $1 / A_{m} \in \mathbb{Z}$, we have $a_{m}=1+\left[1 / A_{m}\right]=1+1 / A_{m}$ and thus

$$
A_{m}=a_{m-1} A_{m-1}-1=n_{m-1} r_{m-2}-1=r_{m-1} .
$$


From the construction of CEF, we know that $n_{m}=\left\lceil 1 / r_{m-1}\right\rceil$. Thus, $n_{m}=1 / A_{m}$ showing that $a_{m}=n_{m}+1$. Furthermore, $r_{m}=n_{m} r_{m-1}-1=0$, implying that the CEF terminates at $n_{m}$, and by Remark (a) after Theorem 2.1, $a_{m}=a_{m+i}(i \geq 1)$.

\section{The Non-Archimedean Case}

We recapitulate some facts about discrete-valued non-archimedean fields taken from [3, Chapter 4]. Let $K$ be a field complete with respect to a discrete non-archimedean valuation $|\cdot|$ and $\mathcal{O}:=\{A \in K ;|A| \leq 1\}$ its ring of integers. The set $D:=\{A \in K ;|A|<1\}$ is an ideal in $\mathcal{O}$ which is both a maximal ideal and a principal ideal generated by a prime element $\pi \in K$. The quotient ring $\mathcal{O} / D$ is a field, called the residue class field. Let $\mathcal{A} \subset \mathcal{O}$ be a set of representatives of $\mathcal{O} / D$. Every $A \in K \backslash\{0\}$ is uniquely of the shape

$$
A=\sum_{n=N}^{\infty} b_{n} \pi^{n} \quad\left(b_{n} \in \mathcal{A}, b_{N} \neq 0\right)
$$

for some $N \in \mathbb{Z}$, and define the $\operatorname{order} v(A)$ of $A$ by $|A|=2^{-v(A)}=2^{-N}$, with $v(0):=+\infty$. The head part $\langle A\rangle$ of $A$ is defined as the finite series

$$
\langle A\rangle=\sum_{n=v(A)}^{0} b_{n} \pi^{n} \quad \text { if } v(A) \leq 0, \text { and } 0 \text { otherwise. }
$$

Denote the set of all head parts by

$$
S:=\{\langle A\rangle ; A \in K\} .
$$

The Knopfmachers' series expansion algorithm for series expansions in $K$ [4] proceeds as follows. For $A \in K$, let

$$
a_{0}:=\langle A\rangle \in S
$$

Define

$$
A_{1}:=A-a_{0}
$$

If $A_{n} \neq 0(n \geq 1)$ is already defined, put

$$
a_{n}=\left\langle\frac{1}{A_{n}}\right\rangle, \quad A_{n+1}=\left(A_{n}-\frac{1}{a_{n}}\right) \frac{s_{n}}{r_{n}}
$$

if $a_{n} \neq 0$, where $r_{n}$ and $s_{n} \in K \backslash\{0\}$ which may depend on $a_{1}, \ldots, a_{n}$. Then for $n \geq 1$

$$
A=a_{0}+A_{1}=\cdots=a_{0}+\frac{1}{a_{1}}+\frac{r_{1}}{s_{1}} \frac{1}{a_{2}}+\cdots+\frac{r_{1} \cdots r_{n-1}}{s_{1} \cdots s_{n-1}} \frac{1}{a_{n}}+\frac{r_{1} \cdots r_{n}}{s_{1} \cdots s_{n}} A_{n+1} .
$$

The process ends in a finite expansion if some $A_{n+1}=0$. If some $a_{n}=0$, then $A_{n+1}$ is not defined. To take care of this difficulty, we impose the condition

$$
v\left(s_{n}\right)-v\left(r_{n}\right) \geq 2 v\left(a_{n}\right)-1 .
$$


Thus

$$
A=a_{0}+\frac{1}{a_{1}}+\sum_{n=1}^{\infty} \frac{r_{1} \cdots r_{n}}{s_{1} \cdots s_{n}} \cdot \frac{1}{a_{n+1}}
$$

When $r_{n}=1, s_{n}=a_{n}$, the algorithm yields a well-defined (with respect to the valuation) and unique series expansion, termed non-archimedean Engel series expansion. Summing up, we have the following.

Theorem 3.1. Every $A \in K \backslash\{0\}$ has a finite or an infinite convergent non-archimedean ES expansion of the form

$$
A=a_{0}+\sum_{n=1}^{\infty} \frac{1}{a_{1} a_{2} \cdots a_{n}}
$$

where the digits $a_{n}$ are subject to the restrictions

$$
a_{0}=\langle A\rangle \in S, \quad a_{n} \in S, \quad v\left(a_{n}\right) \leq-n, \quad v\left(a_{n+1}\right) \leq v\left(a_{n}\right)-1 \quad(n \geq 1) .
$$

Now we turn to the construction of a non-archimedean Cohen-Egyptian fraction expansion, in the same spirit as that of the real numbers, that is, by way of Lemma 2.2. To this end, we start with the following lemma.

Lemma 3.2. For any $y \in K \backslash\{0\}$ such that $v(y) \geq 1$, there exist a unique $n \in S$ such that $v(n) \leq-1$ and a unique $r \in K$ such that

$$
1=n y-r, \quad v(r) \geq v(y)+1 \quad(\text { i.e., } 0 \leq|r|<|y|) .
$$

Proof. Let $n=\langle 1 / y\rangle$. Then $v(n)=v(1 / y)=-v(y) \leq-1$. Putting $r=n y-1$, we show now that $v(r) \geq v(y)+1$. Since $n=\langle 1 / y\rangle$, we have

$$
\frac{1}{y}=n+\sum_{k \geq 1} c_{k} \pi^{k}
$$

where $c_{k} \in \mathcal{A}$, and so

$$
n y-1=-y \sum_{k \geq 1} c_{k} \pi^{k}
$$

Thus

$$
v(r)=v(n y-1)=v(-y)+v\left(\sum_{k \geq 1} c_{k} \pi^{k}\right) \geq v(y)+1>v(y)
$$


To prove the uniqueness, assume that there exist $n_{1} \in S$ such that $v\left(n_{1}\right) \leq-1$ and $r_{1} \in K$ such that

$$
1=n_{1} y-r_{1}, \quad 0 \leq\left|r_{1}\right|<|y|
$$

From $n y-r=1=n_{1} y-r_{1}$, we get $\left(n-n_{1}\right) y=r-r_{1}$. If $n \neq n_{1}$, since $n, n_{1} \in S$ we have $\left|n-n_{1}\right| \geq 1$. Using $|y|>\left|r-r_{1}\right|$, we deduce that

$$
\left|r-r_{1}\right|<|y| \leq\left|n-n_{1}\right||y|=\left|r-r_{1}\right|,
$$

which is a contradiction. Thus, $n=n_{1}$ and so $r=r_{1}$.

For a non-archimedean CEF expansion, we now prove the following.

Theorem 3.3. Each $y \in K \backslash\{0\}$ has a non-archimedean CEF expansion of the form

$$
y=n_{0}+\sum_{k=1}^{\infty} \frac{1}{n_{1} n_{2} \cdots n_{k}}
$$

where

$$
n_{k} \in S, \quad v\left(n_{k}\right) \leq-k, \quad v\left(n_{k+1}\right) \leq v\left(n_{k}\right)-1 \quad(k \geq 1) .
$$

This series representation is unique subject to the digit condition (3.19).

Proof. Define $n_{0}=\langle y\rangle$ and $r_{0}=y-n_{0}$. Then $v\left(r_{0}\right) \geq 1$. If $r_{0}=0$, the process stops and we write $y=n_{0}$. If $r_{0} \neq 0$, by Lemma 3.2, there are $n_{1} \in S$ and $r_{1} \in K$ such that

$$
n_{1}=\left\langle\frac{1}{r_{0}}\right\rangle, \quad r_{1}=n_{1} r_{0}-1,
$$

where $v\left(n_{1}\right) \leq-1$ and $v\left(r_{1}\right) \geq v\left(r_{0}\right)+1$. So

$$
y=n_{0}+r_{0}=n_{0}+\frac{1}{n_{1}}+\frac{r_{1}}{n_{1}} .
$$

If $r_{1}=0$, the process stops and we write $y=n_{0}+1 / n_{1}$. If $r_{1} \neq 0$, by Lemma 3.2, there are $n_{2} \in S$ and $r_{2} \in K$ such that

$$
n_{2}=\left\langle\frac{1}{r_{1}}\right\rangle, \quad r_{2}=n_{2} r_{1}-1,
$$

where $v\left(n_{2}\right) \leq-1$ and $v\left(r_{2}\right) \geq v\left(r_{1}\right)+1$. So

$$
y=n_{0}+\frac{1}{n_{1}}+\frac{1}{n_{1} n_{2}}+\frac{r_{2}}{n_{1} n_{2}} .
$$


International Journal of Mathematics and Mathematical Sciences

Continuing the process, in general,

$$
\begin{gathered}
n_{k}=\left\langle\frac{1}{r_{k-1}}\right\rangle, \quad r_{k}=n_{k} r_{k-1}-1 \\
y=n_{0}+\frac{1}{n_{1}}+\frac{1}{n_{1} n_{2}}+\cdots+\frac{1}{n_{1} n_{2} \cdots n_{k}}+\frac{r_{k}}{n_{1} n_{2} \cdots n_{k}},
\end{gathered}
$$

where

$$
n_{k} \in S, \quad v\left(n_{k}\right) \leq-1, \quad v\left(r_{k}\right) \geq v\left(r_{k-1}\right)+1 \quad(k \geq 1) .
$$

Thus,

$$
v\left(n_{k+1}\right)=-v\left(r_{k}\right) \leq-v\left(r_{k-1}\right)-1=v\left(n_{k}\right)-1 \quad(k \geq 1) .
$$

We observe that the process terminates if $r_{k}=0$. Next, we show that $v\left(n_{k}\right) \leq-k(k \geq 1)$. By construction, we have $v\left(n_{1}\right) \leq-1$. Assume that $v\left(n_{k}\right) \leq-k$, then

$$
v\left(n_{k+1}\right) \leq v\left(n_{k}\right)-1 \leq-k-1 .
$$

Regarding convergence, consider

$$
\begin{aligned}
v\left(\frac{r_{k}}{n_{1} n_{2} \cdots n_{k}}\right) & =-v\left(n_{1}\right)-v\left(n_{2}\right)-\cdots-v\left(n_{k}\right)+v\left(r_{k}\right) \\
& =-v\left(n_{1}\right)-v\left(n_{2}\right)-\cdots-v\left(n_{k}\right)-v\left(n_{k+1}\right) \\
& \geq 1+2+\cdots+k+(k+1) \longrightarrow \infty \quad(k \longrightarrow \infty) .
\end{aligned}
$$

It remains to prove the uniqueness. Suppose that $x \in K \backslash\{0\}$ has two such expansions

$$
x=n_{0}+\sum_{j} \frac{1}{n_{1} n_{2} \cdots n_{j}}=m_{0}+\sum_{i} \frac{1}{m_{1} m_{2} \cdots m_{i}} .
$$

Since $v\left(\sum_{j} 1 / n_{1} n_{2} \cdots n_{j}\right)=v\left(1 / n_{1}\right) \geq 1$ and $n_{0} \in S$, we have $n_{0}=\langle y\rangle$. Similarly, $m_{0}=\langle y\rangle$ yielding by uniqueness $n_{0}=m_{0}$ and $\sum_{j \geq 1} 1 / n_{1} n_{2} \cdots n_{j}=\sum_{i \geq 1} 1 / m_{1} m_{2} \cdots m_{i}$. Putting

$$
\omega:=\sum_{j \geq 1} \frac{1}{n_{1} n_{2} \cdots n_{j}}=\sum_{i \geq 1} \frac{1}{m_{1} m_{2} \cdots m_{i}}
$$


we have $n_{1} \omega=1+\sum_{j \geq 2} 1 / n_{2} \cdots n_{j}$ and so

$$
1=n_{1} \omega-\sum_{j \geq 2} \frac{1}{n_{2} \cdots n_{j}}, \quad v\left(\sum_{j \geq 2} \frac{1}{n_{2} \cdots n_{j}}\right)=v\left(\frac{1}{n_{2}}\right)=-v\left(n_{2}\right) \geq-v\left(n_{1}\right)+1=v(\omega)+1
$$

By Lemma 3.2, since $n_{1}$ is the unique element in $S$ with such property, we deduce $n_{1}=m_{1}$. Continuing in the same manner, we conclude that the two expansions are identical.

It is clear that the construction of non-archimedean ES and CEF expansions is identical which implies at once that the two representations are exactly the same in the nonarchimedean case.

\section{Rationality Characterization in the Non-Archimedean Case}

In the case of real numbers, we have seen that both ES and CEF expansions can be used to characterize rational numbers with quite different outcomes. In the non-archimedean situation, though ES and CEF expansions are identical, their use to characterize rational elements depend significantly on the underlying nature of each specific field. We end this paper by providing information on the rationality characterization in three different nonarchimedean fields, namely, the field of $p$-adic numbers and the two function fields, one completed with respect to the degree valuation and the other with respect to a prime-adic valuation.

The following characterization of rational numbers by $p$-adic ES expansions is due to Grabner and Knopfmacher [5].

Theorem 4.1. Let $x \in p \mathbb{Z}_{p} \backslash\{0\}$. Then $x$ is rational, $x=\alpha / \beta$, if and only if either the $p$-adic ES expansion of $x$ is finite, or there exist an $m$ and an $s \geq m$, such that

$$
a_{m+j}=\frac{p^{s+j+1}-\gamma}{p^{s+j}} \quad(j=0,1,2, \ldots)
$$

where $\gamma \mid \beta$.

Now for function fields, we need more terminology. Let $\mathbb{F}$ denote a field and $\pi(x)$ an irreducible polynomial of degree $d$ over $\mathbb{F}$. There are two types of valuation in the field of rational functions $\mathbb{F}(x)$, namely, the $\pi(x)$-adic valuation $|\cdot|_{\pi}$, and the degree valuation $|\cdot|_{1 / x}$ defined as follows. From the unique representation in $\mathbb{F}(x)$,

$$
\frac{f(x)}{g(x)}=\pi(x)^{m} \frac{r(x)}{s(x)}, \quad f(x), g(x), r(x), s(x) \in \mathbb{F}[x] \backslash\{0\} ; \pi(x) \nmid r(x) s(x), m \in \mathbb{Z},
$$

set

$$
|0|_{\pi}=0, \quad\left|\frac{f(x)}{g(x)}\right|_{\pi}=2^{-m d} ; \quad|0|_{1 / x}=0, \quad\left|\frac{f(x)}{g(x)}\right|_{1 / x}=2^{\operatorname{deg} f(x)-\operatorname{deg} g(x)} .
$$


Let $\mathbb{F}((\pi))$ and $\mathbb{F}((1 / x))$ be the completions of $\mathbb{F}(x)$, with respect to the $\pi(x)$-adic and the degree valuations, respectively. The extension of the valuations to $\mathbb{F}((\pi))$ and $\mathbb{F}((1 / x))$ is also denoted by $|\cdot|_{\pi}$ and $|\cdot|_{1 / x}$.

For a characterization of rational elements, we prove the following.

Theorem 4.2. The CEF of $y \in \mathbb{F}((\pi))$ or in $\mathbb{F}((1 / x))$ terminates if and only if $y \in \mathbb{F}(x)$.

Proof. Although the assertions in both fields $\mathbb{F}((\pi))$ and $\mathbb{F}((1 / x))$ are the same, their respective proofs are different. In fact, when the field $\mathbb{F}$ has finite characteristic, both results have already been shown in [6] and the proof given here is basically the same.

We use the notation of the last section with added subscripts $\pi$ or $1 / x$ to distinguish their corresponding meanings.

If the CEF of $y$ in either field is finite, then $y$ is clearly rational. It remains to prove the converse and we begin with the field $\mathbb{F}((\pi))$. Assume that $y \in \mathbb{F}(x) \backslash\{0\}$. By construction, each $r_{k} \in \mathbb{F}(x)$ and so can be uniquely represented in the form

$$
r_{k}=\pi(x)^{v\left(r_{k}\right)} \frac{p_{k}(x)}{q_{k}(x)}
$$

where $p_{k}(x), q_{k}(x)(\neq 0) \in \mathbb{F}[x]$ with $\operatorname{gcd}\left(p_{k}(x), q_{k}(x)\right)=1, \pi(x) \nmid p_{k}(x) q_{k}(x)$. Since $n_{k}=$ $\left\langle 1 / r_{k-1}\right\rangle \in S_{\pi}$ and $v\left(n_{k}\right) \leq-k$, it is of the form

$$
\begin{aligned}
n_{k} & =s_{v\left(n_{k}\right)}(x) \pi(x)^{v\left(n_{k}\right)}+s_{v\left(n_{k}\right)+1}(x) \pi(x)^{v\left(n_{k}\right)+1}+\cdots+s_{-1}(x) \pi(x)^{-1}+s_{0}(x) \\
& =: m_{k}(x) \pi(x)^{v\left(n_{k}\right)},
\end{aligned}
$$

where $s_{v\left(n_{k}\right)}(x), \ldots, s_{0}(x)$ are polynomials over $\mathbb{F}$, not all 0 , of degree $<d$ and $m_{k}(x) \in \mathbb{F}[x]$. Thus,

$$
\begin{aligned}
\left|n_{k}\right|_{1 / x} & \leq \max \left\{\left|s_{v\left(n_{k}\right)}(x) \pi(x)^{v\left(n_{k}\right)}\right|_{1 / x^{\prime}}\left|s_{v\left(n_{k}\right)+1}(x) \pi(x)^{v\left(n_{k}\right)+1}\right|_{1 / x^{\prime}}, \ldots,\right. \\
& \left.\quad\left|s_{-1}(x) \pi(x)^{-1}\right|_{1 / x^{\prime}}\left|s_{0}(x)\right|_{1 / x}\right\} \\
& \leq 2^{d-1},
\end{aligned}
$$

yielding

$$
\left|m_{k}(x)\right|_{1 / x} \leq 2^{d-d v\left(n_{k}\right)-1} .
$$

By construction, we have

$$
r_{k}=n_{k} r_{k-1}-1
$$


Substituting (4.4) and (4.5) into (4.8) and using $v\left(r_{k-1}\right)=-v\left(n_{k}\right)$ lead to

$$
\pi(x)^{-v\left(n_{k+1}\right)} p_{k}(x) q_{k-1}(x)=q_{k}(x)\left(m_{k}(x) p_{k-1}(x)-q_{k-1}(x)\right) .
$$

Since gcd $\left(\pi(x)^{-v\left(n_{k+1}\right)} p_{k}(x), q_{k}(x)\right)=1$, it follows that $q_{k}(x) \mid q_{k-1}(x)$, and so successively, we have

$$
\left|q_{k}(x)\right|_{1 / x} \leq\left|q_{k-1}(x)\right|_{1 / x} \leq \cdots \leq\left|q_{1}(x)\right|_{1 / x^{\prime}}
$$

which together with (4.9) yield

$$
\left|p_{k}(x)\right|_{1 / x} \leq|\pi(x)|_{1 / x}^{v\left(n_{k+1}\right)} \max \left\{\left|m_{k}(x) p_{k-1}(x)\right|_{1 / x^{\prime}}\left|q_{1}(x)\right|_{1 / x}\right\} .
$$

Using (3.19) and (4.7), we consequently have

$$
\begin{aligned}
\left|p_{k}(x)\right|_{1 / x} & \leq 2^{d\left(v\left(n_{k}\right)-1\right)} \max \left\{2^{d-d v\left(n_{k}\right)-1}\left|p_{k-1}(x)\right|_{1 / x^{\prime}}\left|q_{1}(x)\right|_{1 / x}\right\} \\
& \leq \max \left\{\frac{1}{2}\left|p_{k-1}(x)\right|_{1 / x^{\prime}} \frac{\left|q_{1}(x)\right|_{1 / x}}{2^{d(k+1)}}\right\} .
\end{aligned}
$$

This shows that $\left|p_{k}(x)\right|_{1 / x} \leq(1 / 2)\left|p_{k-1}(x)\right|_{1 / x}$ for all large $k$ which implies that from some $k$ onwards, $p_{k}(x)=0$, and so $r_{k}=0$, that is, the expansion terminates.

Finally for the field $\mathbb{F}((1 / x))$, assume that $y=p(x) / q(x) \in \mathbb{F}(x) \backslash\{0\}$. Without loss of generality, assume $\operatorname{deg} p(x) \geq \operatorname{deg} q(x)$. By the Euclidean algorithm, we have

$$
y=\frac{p(x)}{q(x)}=N_{0}(x)+\frac{R_{0}(x)}{q(x)}:=n_{0}+r_{0}
$$

where

$$
n_{0}:=N_{0}(x)=\langle y\rangle_{1 / x^{\prime}} \quad R_{0}(x) \in \mathbb{F}[x], \quad 0 \leq \operatorname{deg} R_{0}<\operatorname{deg} q, \quad r_{0}=\frac{R_{0}(x)}{q(x)}
$$

From the Euclidean algorithm,

$$
\frac{q(x)}{R_{0}(x)}=N_{1}(x)+\frac{R_{1}(x)}{R_{0}(x)}, \quad N_{1}(x), R_{1}(x) \in \mathbb{F}[x], \quad 0 \leq \operatorname{deg} R_{1}<\operatorname{deg} R_{0}<\operatorname{deg} q,
$$

which is, in the terminology of Lemma 3.2,

$$
1=r_{0} N_{1}+\frac{R_{1}}{q}=r_{0} n_{1}-r_{1}
$$


Again, from the Euclidean algorithm,

$$
\frac{-q(x)}{R_{1}(x)}=N_{2}(x)+\frac{R_{2}(x)}{R_{1}(x)}, \quad N_{2}(x), R_{2}(x) \in \mathbb{F}[x], \quad 0 \leq \operatorname{deg} R_{2}<\operatorname{deg} R_{1}<\operatorname{deg} R_{0}<\operatorname{deg} q,
$$

which is, in the terminology of Lemma 3.2,

$$
1=r_{1} N_{2}-\frac{R_{2}}{q}=r_{1} n_{2}-r_{2}
$$

Proceeding in the same manner, in general we have

$$
r_{j}=(-1)^{j} \frac{R_{j}}{q}, \quad 0 \leq \operatorname{deg} R_{j}<\operatorname{deg} R_{j-1}<\cdots<\operatorname{deg} R_{1}<\operatorname{deg} q .
$$

There must then exist $k \in \mathbb{N}$ such that $\operatorname{deg} R_{k}=0$, that is, $R_{k} \in \mathbb{F} \backslash\{0\}$. Thus, the CEF of $y$ is

$$
y=n_{0}+\frac{1}{n_{1}}+\cdots+\frac{1}{n_{1} \cdots n_{k}}+\frac{r_{k}}{n_{1} \cdots n_{k}}=n_{0}+\frac{1}{n_{1}}+\cdots+\frac{1}{n_{1} \cdots n_{k}}+\frac{1}{n_{1} \cdots n_{k} n_{k+1}},
$$

where $n_{k+1}=(-1)^{k} R_{k}^{-1} q \in \mathbb{F}[x]$, which is a terminating CEF.

\section{Acknowledgments}

This work was supported by the Commission on Higher Education and the Thailand Research Fund RTA5180005 and by the Centre of Excellence In Mathematics, the Commission on Higher Education.

\section{References}

[1] O. Perron, Irrationalzahlen, Chelsea, New York, NY, USA, 1951.

[2] R. Cohen, "Egyptian fraction expansions," Mathematics Magazine, vol. 46, pp. 76-80, 1973.

[3] J. W. S. Cassels, Local Fields, vol. 3 of London Mathematical Society Student Texts, Cambridge University Press, Cambridge, UK, 1986.

[4] A. Knopfmacher and J. Knopfmacher, "Series expansions in p-adic and other non-archimedean fields," Journal of Number Theory, vol. 32, no. 3, pp. 297-306, 1989.

[5] P. J. Grabner and A. Knopfmacher, "Arithmetic and metric properties of $p$-adic Engel series expansions," Publicationes Mathematicae Debrecen, vol. 63, no. 3, pp. 363-377, 2003.

[6] V. Laohakosol, N. Rompurk, and A. Harnchoowong, "Characterizing rational elements using Knopfmachers' expansions in function fields," Thai Journal of Mathematics, vol. 4, no. 1, pp. 223-244, 2006. 


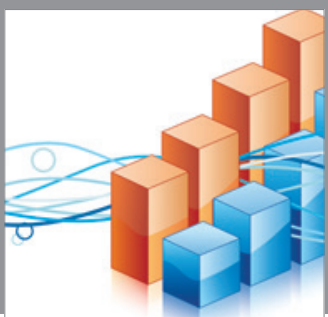

Advances in

Operations Research

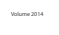

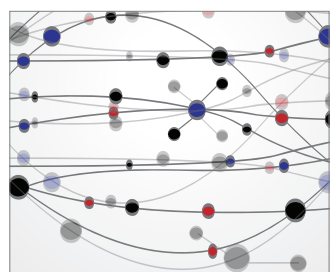

\section{The Scientific} World Journal
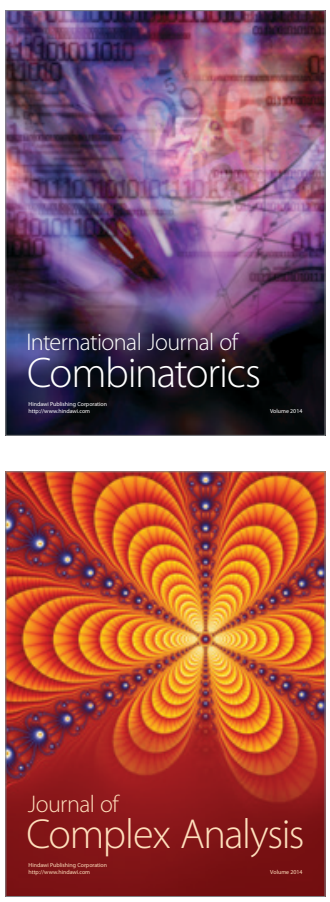

International Journal of

Mathematics and

Mathematical

Sciences
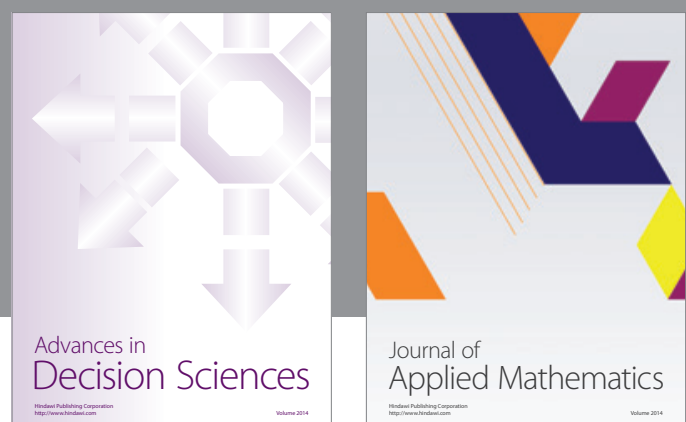

Journal of

Applied Mathematics
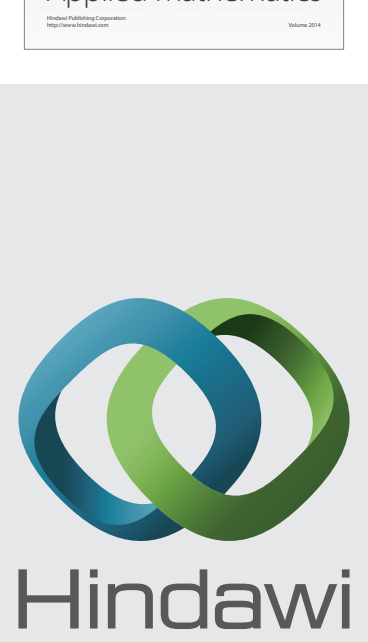

Submit your manuscripts at http://www.hindawi.com
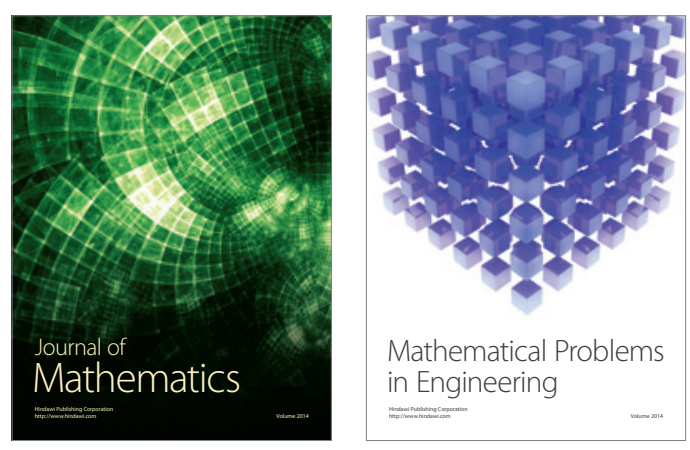

Mathematical Problems in Engineering
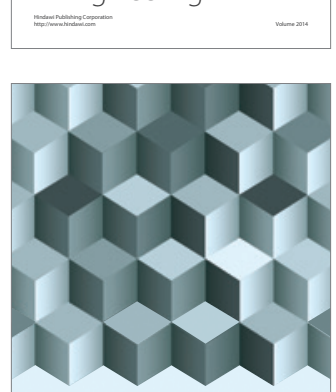

Journal of

Function Spaces
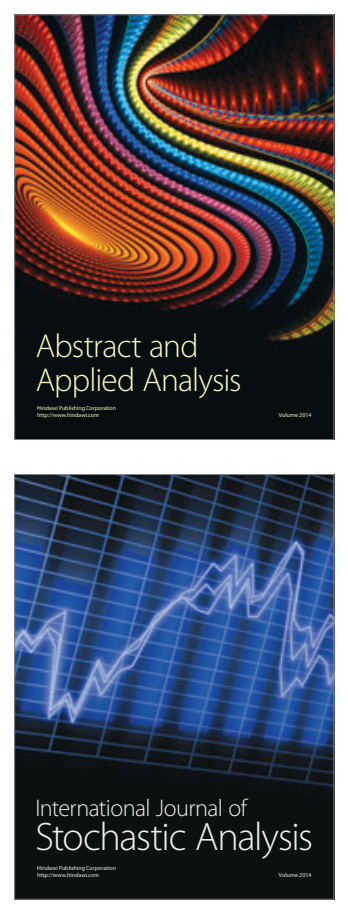

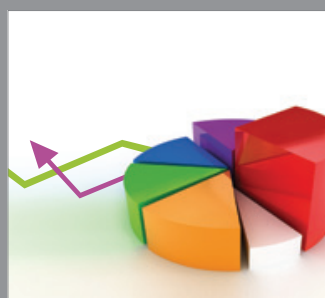

ournal of

Probability and Statistics

Promensencen
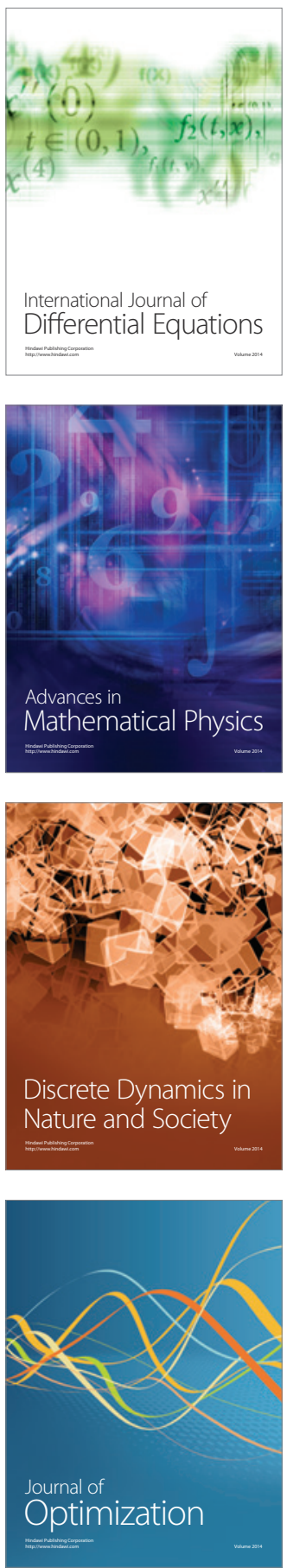